\title{
The auroral radio emission of the magnetic B-type star $\rho$ OphC
}

\author{
P. Leto,${ }^{1}{ }^{\star}$ C. Trigilio,,${ }^{1}$ C. S. Buemi,${ }^{1}$ F. Leone,${ }^{2}$ I. Pillitteri,${ }^{3}$ L. Fossati,${ }^{4}$ F. Cavallaro, ${ }^{1}$ \\ L. M. Oskinova, ${ }^{5,6}$ R. Ignace,${ }^{7}$ J. Krtička,${ }^{8}$ G. Umana, ${ }^{1}$ G. Catanzaro, ${ }^{1}$ A. Ingallinera, ${ }^{1}$ \\ F. Bufano, ${ }^{1}$ S. Riggi, ${ }^{1}$ L. Cerrigone, ${ }^{9}$ S. Loru, ${ }^{1}$ F. Schilliró,${ }^{1}$ C. Agliozzo, ${ }^{10}$ \\ N. M. Phillips, ${ }^{10}$ M. Giarrusso, ${ }^{11}$ J. Robrade ${ }^{12}$ \\ ${ }^{1}$ INAF - Osservatorio Astrofisico di Catania, Via S. Sofia 78, 95123 Catania, Italy \\ ${ }^{2}$ Universitá di Catania, Dipartimento di Fisica e Astronomia, Sezione Astrofisica, Via S. Sofia 78, I-95123 Catania, Italy \\ ${ }^{3}$ INAF - Osservatorio Astronomico di Palermo, Piazza del Parlamento 1, 90134 Palermo, Italy \\ ${ }^{4}$ Space Research Institute, Austrian Academy of Sciences, Schmiedlstrasse 6, A-8042 Graz, Austria \\ ${ }^{5}$ Institute for Physics and Astronomy, University Potsdam, 14476 Potsdam, Germany \\ ${ }^{6}$ Kazan Federal University, Kremlevskaya Str 18, Kazan, Russia \\ ${ }^{7}$ Department of Physics \& Astronomy, East Tennessee State University, Johnson City, TN 37614, USA \\ ${ }^{8}$ Department of Theoretical Physics and Astrophysics, Masaryk University, Kotlářská 2, CZ-611 37 Brno, Czech Republic \\ ${ }^{9}$ Joint ALMA Observatory, Alonso de Córdova 3107, Vitacura, Santiago, Chile \\ ${ }^{10}$ European Southern Observatory, Karl-Schwarzschild-Strasse 2, Garching bei München, 85748, Germany \\ ${ }^{11}$ INFN, Laboratori Nazionali del Sud, Via S. Sofia 62, I-95123 Catania, Italy \\ ${ }^{12}$ Hamburger Sternwarte, University of Hamburg, Gojenbergsweg 112, D-21029 Hamburg, Germany
}

\begin{abstract}
The non-thermal radio emission of main-sequence early-type stars is a signature of stellar magnetism. We present multi-wavelength (1.6-16.7 GHz) ATCA measurements of the earlytype magnetic star $\rho \mathrm{OphC}$, which is a flat-spectrum non-thermal radio source. The $\rho$ OphC radio emission is partially circularly polarized with a steep spectral dependence: the fraction of polarized emission is about $60 \%$ at the lowest frequency sub-band $(1.6 \mathrm{GHz})$ while is undetected at $16.7 \mathrm{GHz}$. This is clear evidence of coherent Auroral Radio Emission (ARE) from the $\rho$ OphC magnetosphere. Interestingly, the detection of the $\rho$ OphC's ARE is not related to a peculiar rotational phase. This is a consequence of the stellar geometry, which makes the strongly anisotropic radiation beam of the amplified radiation always pointed towards Earth. The circular polarization sign evidences mainly amplification of the ordinary mode of the electromagnetic wave, consistent with a maser amplification occurring within dense regions. This is indirect evidence of the plasma evaporation from the polar caps, a phenomenon responsible for the thermal $\mathrm{X}$-ray aurorae. $\rho \mathrm{OphC}$ is not the first early-type magnetic star showing the $\mathrm{O}$-mode dominated ARE but is the first star with the ARE always on view.
\end{abstract}

Key words: masers - stars: early-type - stars: individual: $\rho$ OphA - stars: magnetic field radio continuum: stars $-\mathrm{X}$-rays: stars.

\section{INTRODUCTION}

Magnetic early-type (OBA) stars show a typical rotational variability observed in many bands of the electromagnetic spectrum, from the UV (Shore \& Adelman 1981) to the radio domain (Leone 1991). Such rotational modulation is well explained in the framework of the Oblique Rotator Model (ORM), where the overall stellar magnetic field topology is described by an almost dipolar field (at $\mathrm{kG}$ level) with the magnetic axis not-aligned with the stellar rotation axis (Babcock 1949; Stibbs 1950).

About $25 \%$ of the known magnetic early-type stars are non-

* E-mail: paolo.leto@inaf.it

(c) The Authors thermal radio sources (Drake et al. 1987; Linsky, Drake \& Bastian 1992; Leone, Trigilio \& Umana 1994). In many cases such kind of stars are also X-ray sources (Nazé et al. 2014). The main observed features, from X-ray to radio wavelengths, are explained within a common framework outlined by the magnetically confined wind shock (MCWS) model (Babel \& Montmerle 1997). Inside the "inner magnetosphere" the magnetic field traps and channels the ionized matter. The wind plasma streams from opposites hemispheres are then forced to move along the dipolar magnetic field lines. At the magnetic equator the streams collide and shock, radiating thermal X-rays. Far from the star, the magnetic field is no longer able to confine the plasma. In the resulting current sheets, electrons can be accelerated up to relativistic energies. These non-thermal electrons 
move within the "middle magnetosphere" and radiate through incoherent gyro-synchrotron mechanism. The above scenario has been confirmed by a 3D modeling approach, where the radiative transfer equation for the gyro-synchrotron emission mechanism has been integrated along the line of sight producing the synthetic brightness spatial distribution and total flux from the co-rotating stellar magnetosphere (Trigilio et al. 2004; Leto et al. 2006).

Among the class of the early-type magnetic stars, six are known to behave like radio pulsars (Trigilio et al. 2000; Das, Chandra \& Wade 2018; Das et al. 2019a,b; Leto et al. 2019, 2020). These six sources show low frequency highly polarized pulses, explained as the stellar analog of the coherent Auroral Radio Emission (ARE) observed on the magnetized planets of the solar system (Zarka 1998). The ARE arises from magnetospheric regions above the auroral ovals, located at the planetary surface, and is observed from infrared to X-ray bands (Badman et al. 2015).

The amplification mechanism of the ARE is the Electron Cyclotron Maser (ECM) powered by an unstable energy distribution, which can be developed by the electrons moving within a magnetospheric cavity (Wu \& Lee 1979; Melrose \& Dulk 1982). The ECM emission mechanism amplifies the radiation at frequencies close to the first few harmonics of the local gyro-frequency $v_{\mathrm{B}}=$ $2.8 \times 10^{-3} \mathrm{~B} / \mathrm{G} \mathrm{GHz}$, hence the frequency of the ARE is directly related to the local magnetic field strength. The ECM mainly amplifies just one of the two magneto-ionic modes (each one with opposite circular polarization direction), resulting in the high polarization degree of the ARE. The mainly amplified magneto-ionic mode depends on the density of local thermal plasma (Sharma \& Vlahos 1984; Melrose, Hewitt \& Dulk 1984).

The ECM theory predicts that the amplified radiation is constrained within a strongly anisotropic beam shaped like a thin hollow cone of large half-aperture $\left(\approx 90^{\circ}\right)$ and centered to the magnetic field line. The ECM ray path is therefore oriented almost perpendicularly with respect to the local magnetic field vector, making the ARE observable only when the magnetic field vectors of the auroral source regions are almost perpendicular to the line of sight. The ARE from the early-type magnetic stars is produced in thin dipole shaped magnetic cavity, the middle-magnetosphere. The elementary ECM sources aligned along the direction that maximizes the path inside the thin auroral cavity coherently contribute to produce the detectable ARE. Hence, in case of ECM amplified within a thin magnetic shell, the overall ARE beam is mainly oriented along the plane tangent to the auroral cavity wall, like the case of the Earth's auroral kilometric radiation (Louarn \& Le Queau 1996). Interestingly, in some early-type magnetic stars the signature of the auroral emission was discovered in the X-ray spectra (Leto et al. 2017, 2018, 2020; Robrade et al. 2018), and in the case of $\rho$ OphA also in its X-ray light curve (Pillitteri et al. 2017).

In this paper we present new multi-wavelength radio measurements of $\rho$ OphC (HD 147932), a magnetic (Alecian et al. 2014) B5V type star that has also exhibits X-ray pulses (Pillitteri et al. 2016), like the case of $\rho$ OphA (Pillitteri et al. 2017). $\rho$ OphC exhibits coherent and incoherent radio emission, whose study allowed us to characterize its magnetosphere. The radio and X-ray combined results allowed us to better understand the plasma processes occurring within the stellar magnetosphere.

\section{RADIO MEASUREMENTS}

New L, C, X, and U-bands radio observations (centered at 2.1, 5.5, 9, and $16.7 \mathrm{GHz}$ ) of $\rho$ OphC were performed on 2019 March by the Australian Telescope Compact Array ${ }^{1}$ (ATCA). The data were edited and calibrated using the standard MIRIAD software package. The backend system has a bandwidth $2 \mathrm{GHz}$ wide, for each band, which allows us to better investigate the spectral dependence of the $\rho$ OphC's radio emission by analyzing the $1 \mathrm{GHz}$ wide sub-bands.

$\rho$ OphC was observed by ATCA during three epochs, about $10 \mathrm{hrs}$ per epoch, when the interferometer was pointing towards $\rho$ OphA (data already published, see Leto et al. 2020 for further technical details). Hence $\rho$ OphC was observed $\approx 2.5^{\prime}$ away from the phase tracking center. To obtain reliable flux density measurements, we performed the primary beam correction using the task LINMOS. The correction to apply increases with the observing frequency, as a consequence the noise background level also increases. The U-band measurements were performed using the $15 \mathrm{~mm}$ receiver that acquires 2 simultaneous bands at 16.7 (U-band) and 21.2 $\mathrm{GHz}$ (K-band). Unfortunately $\rho \mathrm{OphC}$ is close to the primary beam first null at the K-band, hence we were not able to retrieve the highest frequency band of the $15 \mathrm{~mm}$ receiver.

The ATCA is a linear interferometer that precludes making maps with short integration time (source snapshots), therefore, we retrieved the time dependent flux density measurements of $\rho \mathrm{OphC}$, both for the Stokes $I$ and $V$, by means of the discrete Fourier transform of the complex visibilities at the source position (Trigilio et al. 2008, 2018; Leto et al. 2020). No sources are present around the field centered on $\rho \mathrm{OphC}$ (the closest radio source is $\rho \mathrm{OphA}$ ) enabling us to rely on the time resolved measurements.

\section{THE RADIO EMISSION OF $\rho$ OphC}

To search for possible rotational modulation of the radio emission of $\rho \mathrm{OphC}$, we phase folded the time resolved multi-wavelength ATCA measurements. The photometric time series provided by the Kepler's K2 mission clearly evidence the rotation period of $P=0.8639$ days (Rebull et al. 2018). The phase folded ATCA observations uniformly sample the rotational period. The zero phase is the start of the ATCA observations: HJD $=2458555.073$.

The wide band radio emission of $\rho \mathrm{OphC}$ is roughly modulated by the stellar rotation, see Fig. 1, similar to other magnetic early-type stars well studied at the radio band (Trigilio et al. 2004; Leto et al. 2006, 2012, 2017, 2018, 2020; Bailey et al. 2012). We also observed large variation between measurements performed at similar phases, this suggests that some other kind of variability mechanism overlaps with the rotational modulation.

To better analyze the radio spectrum the ATCA measurements at the $\mathrm{L}$ and $\mathrm{C}$ bands are splitted each in two sub-bands $1 \mathrm{GHz}$ wide (centered at $v=1.6$ and $2.6 \mathrm{GHz}$ for the $\mathrm{L}$ band; at $v=5$ and $6 \mathrm{GHz}$ for the $\mathrm{C}$ band). The corresponding total intensities, average of the time resolved radio measurements of all the epochs, are shown in the top panel of Fig. 2. The flux of $\rho$ OphC at the U-band ( $v=16.7$ $\mathrm{GHz}$ ) is $S_{\mathrm{I}}=20.7 \pm 0.2 \mathrm{mJy}$, this value was measured from the $\mathrm{U}$ band radio map performed collecting all the observing epochs, the corresponding $S_{\mathrm{V}}$ is below the detection threshold.

The average radio spectrum of $\rho \mathrm{OphC}$ is quite flat with a positive spectral index (Fig. 2 top panel). The average total flux density (frequency range $1.6-16.7 \mathrm{GHz}$ ) is $\left\langle S_{\mathrm{I}}\right\rangle \approx 18 \mathrm{mJy}$. Then, at the distance $d=134$ pc (Gaia Collaboration 2018), the spectral radio

1 The Australia Telescope Compact Array is part of the Australia Telescope National Facility which is funded by the Australian Government for operation as a National Facility managed by CSIRO 

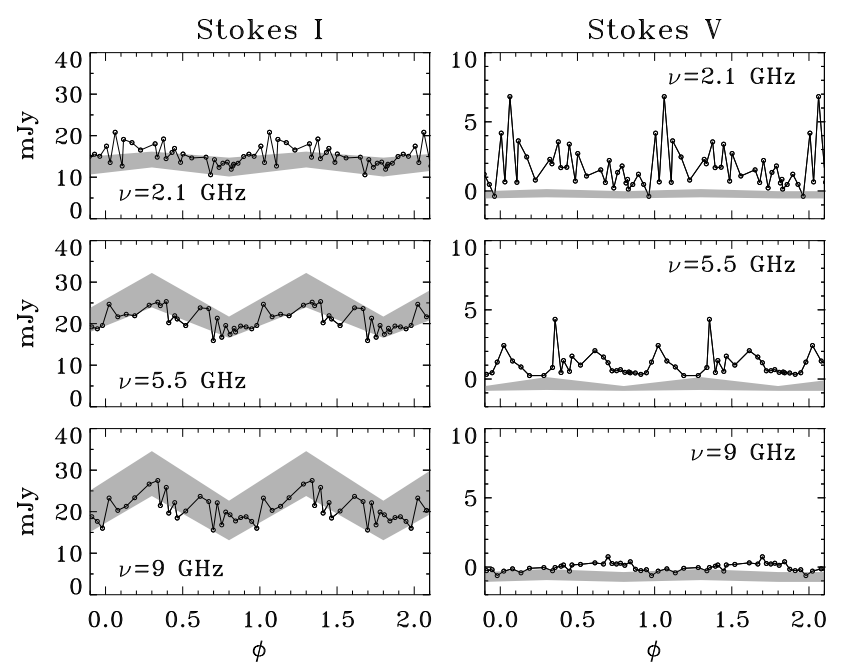

Figure 1. Left panels: measured multi-wavelengths phase-folded light curves of the total intensity (Stokes $I$ ) of $\rho$ OphC (integration time $\approx$ 15 minutes). Right panels: light curves of the circularly polarized emission (Stokes $V$ ). The gray regions show the theoretical incoherent gyrosynchrotron emission (both for the Stokes $I$ and $V$ ) simulated using the stellar parameters of $\rho$ OphC.

luminosity of $\rho$ OphC is $L_{v \text {, rad }} \approx 4 \times 10^{17} \mathrm{erg} \mathrm{s}^{-1} \mathrm{~Hz}^{-1}$. Luminosity level and flat spectrum are absolutely normal for such kind of magnetic stars (Leto et al. 2017, 2018, 2020).

The $\rho$ OphC low frequency emission is circularly polarized, with the polarization level that steeply decreases as the observing radio frequency increases. Figure 2 (bottom panel) shows the spectral dependence of the measured range of the circular polarization fraction $\pi_{\mathrm{c}}\left(S_{\mathrm{V}} / S_{\mathrm{I}}\right)$. The sub-band centered at $1.6 \mathrm{GHz}$ evidence the highest level of circularly polarized emission, in particular the highest measured level was $\pi_{\mathrm{c}} \approx 60 \%$. The Right hand Circularly Polarized (RCP) emission is dominant (Stokes $V>0$ ). Such high level of circular polarization of the low frequency emission has already been observed in six other early-type magnetic stars. Acting as radio lighthouses, these stars present highly polarized radio pulses. Such behavior has been explained as stellar auroral radio emission (Trigilio et al. 2011) powered by the ECM coherent emission mechanism. The observed high circular polarization level suggests that the magnetosphere of $\rho \mathrm{OphC}$ is also a source of coherent auroral radio emission. However, unlike the other magnetic stars, the detection of the ARE from $\rho$ OphC does not seem to be related to a particular stellar rotational phase. Looking at the right panels of Fig. 1, it is evident that the $\rho$ OphC polarized emission is not constrained to specific phase ranges, as opposed to the coherent pulses of the other six early-type magnetic stars sources of stellar ARE, that are observed at well defined phases.

\section{RADIO EMISSION MODELING}

\subsection{Coherent auroral emission}

To further investigate the nature of the low frequency emission mechanism operating in $\rho$ OphC, we performed an extensive set of simulations of the auroral radio emission visibility from a dipole shaped stellar magnetosphere (see Leto et al. 2016 for details). The ARE beam pattern is strongly anisotropic, this is defined by the hollow-cone half aperture $\left(\theta_{\mathrm{B}}\right)$ and thickness $(\Delta \theta)$, and by the opening angle $(\delta)$ of the radiation diagram centered on the plane tangent to the auroral cavity.
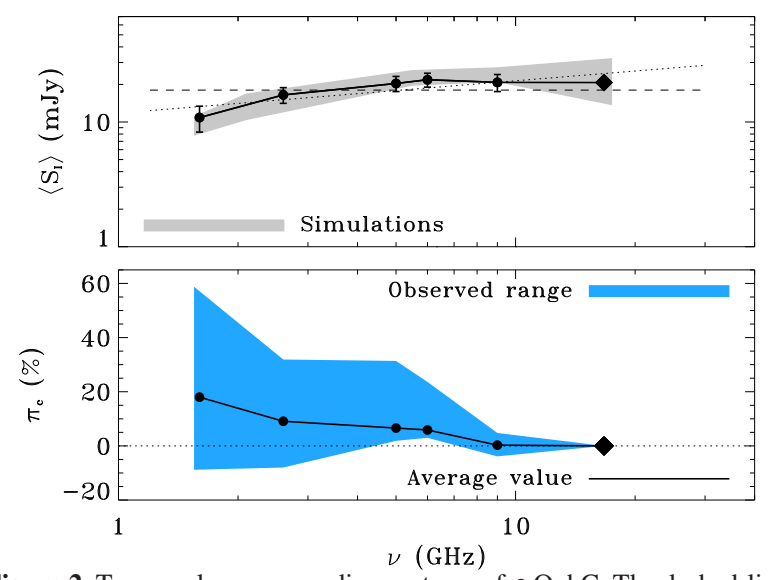

Figure 2. Top panel: average radio spectrum of $\rho$ OphC. The dashed-line is a perfectly flat spectrum, the dotted-line is the fit performed using a powerlaw. The gray area is the envelop of the simulated spectra performed using different model parameters. Bottom panel: spectrum of the fraction of the circularly polarized emission. The thick solid line show $\pi_{\mathrm{c}}$ averaged over the whole rotation period. The blue area locates the $\pi_{\mathrm{c}}$ extrema measured at the different observing frequencies. The diamond symbol in both panels refers to the U-band measurement.

X-ray variability of $\rho$ OphC closely resembles the case of $\rho$ OphA. In case of $\rho$ OphA this variability was recognized as signature of the X-ray counterpart of the ARE. The signature of X-ray auroral emission was also attributed to the hottest thermal component $(>40 \mathrm{MK})$ needed to fit the X-ray spectrum of $\rho$ OphA. Also in case of $\rho$ OphC the X-ray spectral model fitting suggests the presence of gas at temperature $\approx 60 \mathrm{MK}$ (taken from Table D. 1 of Pillitteri et al. 2016). Similarly to $\rho$ OphA, which has its X-ray emission mainly sustained by auroral emission (Leto et al. 2020), the high temperature plasma component in the $\rho$ OphC's X-ray spectrum is likely produced by those relativistic electrons responsible of all the features observed at radio wavelengths. The above considerations can be used to constraint the ARE beam parameters. According to the ECM theory the angles $\theta_{\mathrm{B}}$ and $\Delta \theta$ are related to the radiating electrons speed $(v): \theta=\arccos v / c ; \Delta \theta \approx v / c(c$ is the speed of light). For electrons with energy $\approx 5 \mathrm{keV}$ ( $T \approx 60 \mathrm{MK})$, it follows: $\theta_{\mathrm{B}} \approx 82^{\circ} ; \Delta \theta \approx 8^{\circ}$.

Because of the high ARE directivity, the ORM geometry is crucial for the auroral emission detection. We derived the inclination of the stellar rotation axis (angle $i$ ) of $\rho$ OphC using the relation $v \sin i=2 \pi R_{*} \sin i / P_{\text {rot }}$. Adopting the projected rotational velocity $v \sin i=186 \mathrm{~km} \mathrm{~s}^{-1}$ (Jilinski et al. 2006) and the stellar radius $R_{*}=3.3 \mathrm{R}_{\odot}\left(\right.$ Gaia Collaboration 2018), it follows $i \approx 74^{\circ} . \rho$ OphC is a magnetic star, two longitudinal average magnetic field measurements $\left(\left\langle B_{\mathrm{Z}}\right\rangle\right)$, separated by one day, were reported by Alecian et al. (2014). The $\left\langle B_{\mathrm{Z}}\right\rangle$ values are both negative and with comparable strength $(\approx-1 \mathrm{kG})$. The magnetic field axis orientation (angle $\beta$ between magnetic and rotation axes) has been varied in the range $0^{\circ}<\beta<10^{\circ}$. Larger values of $\beta$ produce large $\left\langle B_{\mathrm{Z}}\right\rangle$ rotational modulation unable to reproduce the almost constant $\left\langle B_{\mathrm{z}}\right\rangle$ measurements. The corresponding polar magnetic field strength $\left(B_{\mathrm{p}}\right)$ lies in the range $12-15.8 \mathrm{kG}$. The other free parameter is the tangent plane opening angle $\delta$, that was linearly increased from $5^{\circ}$ up to $60^{\circ}$ (step $1^{\circ}$ ). Results of our simulations are shown in Fig. 3. We found that, once fixed $\beta$, there are values of $\delta$ able to produce ARE visible for a large fraction of the stellar rotation. The sources of detectable ECM are mainly located in the southern hemisphere, but visible sources of ECM located in the northern hemisphere exist, this reduces the polarization level of the measured ARE. 


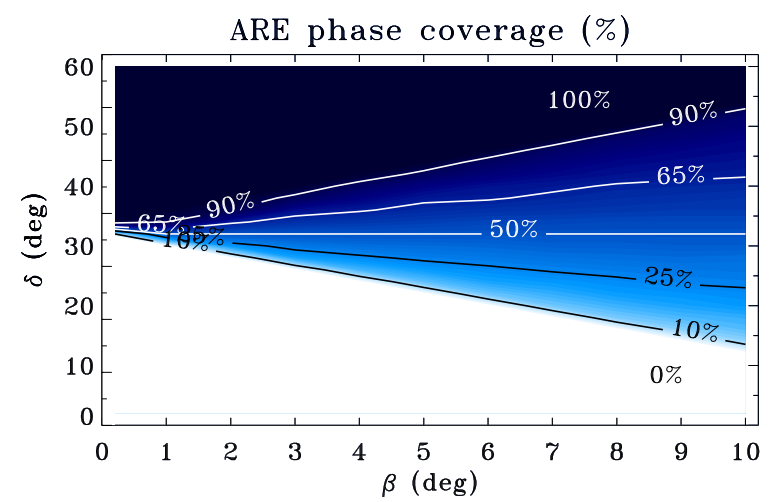

Figure 3. Phase coverage of the ARE from $\rho$ OphC simulated varying the parameters $\beta$ and $\delta$. The dark blue region locates the parameter combinations that make the ARE always detectable. The parameter combinations in the white area precludes the ARE detection.

\subsection{Incoherent gyro-synchrotron emission}

Once constrained the ORM geometry of $\rho$ OphC, we simulated its incoherent gyro-synchrotron emission adopting the central values derived in Sec. $4.1, \beta=5^{\circ}$ and $B_{\mathrm{p}}=13.2 \mathrm{kG}$. Our 3D model is able to simulate the brightness spatial distribution and the total flux produced by a population of non-thermal electrons trapped in the middle magnetosphere. Following the MCWS scenario, the inner magnetosphere is filled by thermal electrons with a temperature that increases linearly outward, starting from the stellar temperature $T_{\text {eff }}=17 \pm 1 \mathrm{kK}$ (Alecian et al. 2014). Their number density is instead inversely related to the radial distance. We performed simulations varying the electrons density at the stellar surface $\left(n_{0}\right)$ in the range $10^{7}-10^{10} \mathrm{~cm}^{-3}$. The non-thermal electrons are powerlaw energy distributed $\left(E^{-p}\right)$ with the index $p$ in the range 2-4.

The equatorial extension of the middle magnetosphere is given by the Alfvén radius $\left(R_{\mathrm{A}}\right)$, that locates the distance at the magnetic equatorial plane where the plasma pressure equates the magnetic energy density. Outside the Alfvén radius the magnetic field strength will be unable to force the plasma to co-rotate with the star, this is the acceleration site of the non-thermal electrons. We varied $R_{\mathrm{A}}$ in the range $10-30 \mathrm{R}_{*}$ (step $5 \mathrm{R}_{*}$ ). The linear size of the non-thermal electrons acceleration region, corresponding to the equatorial thickness $(l)$ of the middle-magnetosphere, and the relativistic electrons density $\left(n_{\mathrm{r}}\right)$ are degenerate parameters. Hence, we varied the column density $n_{\mathrm{r}} \times l$ of the non-thermal electrons located at $R_{\mathrm{A}}$.

Among the full range of the explored model parameters, we found some sets of parameters able to roughly reproduce the observed rotational modulation and spectrum for the total intensity radio emission. The simulated light curves (both for the Stokes $I$ and $V$ ) and the corresponding average spectra are shown in Figs. 1 and 2 (gray regions). Model solutions are obtained using $R_{\mathrm{A}}=15$ $\mathrm{R}_{*}$ and $n_{0}=1-3 \times 10^{9} \mathrm{~cm}^{-3}$. The column density $n_{\mathrm{r}} \times l$ lies in the range $10^{14.9}-10^{17.6} \mathrm{~cm}^{-2}$, as $p$ increases from 2 to 4 . This is a consequence of the highest energy electrons decay when the energy spectrum becomes steeper.

Conversely to the Stokes $I$ (left panels of Fig. 1), the Stokes $V$ simulated level of the incoherent gyro-synchrotron emission is far from the measured level of the $\rho \mathrm{OphC}$ polarized emission, mostly at the lower frequencies (see right panels of Fig. 1). This is further evidence that an additional emission mechanism is in act within the magnetosphere of $\rho \mathrm{OphC}$, namely the coherent ARE.

\section{RESULTS}

The $\rho$ OphC's magnetosphere is viewed almost equator on with the southern magnetic pole always visible. The simulations of the ARE visibility locate the sources of detectable coherent emission mainly in the southern magnetic hemisphere. Furthermore, the observed circular polarization sense is mainly RCP. These two evidences are in accordance with the ECM mechanism that amplifies the ordinary magneto ionic mode within the $\rho$ OphC's magnetosphere, like observed in other two early-type magnetic stars (Leto et al. 2019; Das et al. 2019b).

The ECM coherent emission mechanism mainly amplifies the O-mode when the condition $v_{\mathrm{p}} / v_{\mathrm{B}}>0.3-0.35$ (with $v_{\mathrm{p}}$ the local plasma frequency) is satisfied (Sharma \& Vlahos 1984; Melrose et al. 1984). The plasma frequency is related to the thermal plasma density $\left(N_{\mathrm{e}}\right)$ as $v_{\mathrm{p}}=9 \times 10^{-6} \sqrt{N}_{\mathrm{e}}(\mathrm{GHz})$. The above condition can be used to constrain the density of the local thermal plasma. If the first harmonic of the local gyro-frequency amplified by the ECM mechanism is able to escape, the ARE of $\rho$ OphC at $v=1.6 \mathrm{GHz}$ will arise from magnetospheric regions at height $\approx 1.8 \mathrm{R}_{*}$ above the magnetic pole. If we observed the second harmonic of $v_{\mathrm{B}}$, that is more likely due to the strong gyro-magnetic absorption effects suffered by the $1^{\text {st }}$ harmonic radiation, the ARE emitting region will be located at $\approx 2.6 \mathrm{R}_{*}$. The corresponding electron density needed to produce O-mode amplification for the second harmonic radiation will be $N_{\mathrm{e}}>9.7 \times 10^{8} \mathrm{~cm}^{-3}$.

$\rho \mathrm{OphC}$ is surrounded by a large magnetosphere, which emits at the radio regime for the incoherent gyro-synchrotron emission mechanism and the coherent ECM emission mechanism. The scenario able to summarize the observed radio features of $\rho \mathrm{OphC}$ is pictured in Fig. 4. Following the modeling approach described in Sec. 4.2 we performed the synthetic brightness spatial distribution of the $\rho$ OphC incoherent gyro-synchrotron emission at $v=5.5$ GHz. In Fig. 4, the simulated radio map are shown using the green color levels scaled to the highest brightness value. As additional result of the analysis performed in Sec. 4.1, the simulated map of the ARE has been performed. The blue bright regions pictured in Fig. 4 above the south pole locate the source of coherent emission.

The fast electrons, responsible of the gyro-synchrotron emission, impinging on the surface at the polar caps will be lost. Hence, the non-thermal electrons reflected by the magnetic mirroring mechanism will be deprived by the electrons that impact the stellar surface. This is a suitable condition for the onset of the unstable loss-cone electrons energy distribution, that drives the elementary ECM coherent emission mechanism able to power the observed auroral radio emission from $\rho$ OphC. Meantime, the fast electrons impacting with the stellar surface might produce plasma evaporation enhancing the thermal electron density of the middlemagnetosphere close to the star. The increased plasma density explains the O-mode maser amplification.

The evaporating hot thermal plasma might be also source of thermal auroral X-rays. This X-ray source is located within the red box pictured in Fig. 4 . The emission measure $\left(E M=N_{\mathrm{e}}^{2} V\right)$ of the $\mathrm{X}$-ray source is constrained by using the plasma density needed to detect the O-mode of the coherent ARE. The source volume $(V)$ is instead constrained by using the model parameters that reproduce the incoherent gyro-synchrotron radio emission of $\rho$ OphC (Sec. 4.2). The size of the middle-magnetosphere is $R_{\mathrm{A}}=15 \mathrm{R}_{*}$, the equatorial thickness $(l)$ of the non-thermal acceleration region was between $0.1-1 R_{\mathrm{A}}$. As previously discussed $l$ cannot be univocally assigned, therefore we estimated the $E M$ lower limit in the range $2-10 \times 10^{52} \mathrm{~cm}^{-3}$, roughly assuming a homogeneous plasma 


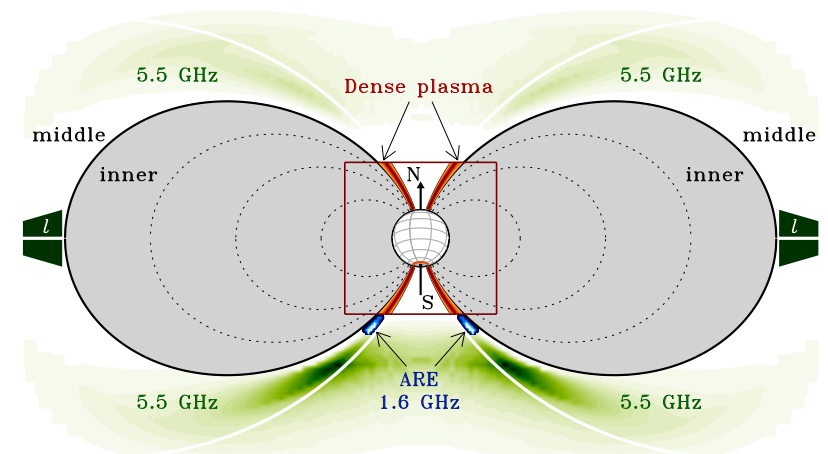

Figure 4. Simulated radio map at $v=5.5 \mathrm{GHz}$ (green) of the $\rho$ OphC incoherent gyro-synchrotron emission. A cartoon showing the different regions of the $\rho$ OphC magnetosphere was superimposed to the synthetic radio map. The grey area pictures the inner magnetosphere where the magnetic field strength is high enough to confine the thermal plasma. The middle magnetosphere is located outside and is delimited by the white thick lines. The blue bright spots locate the magnetospheric regions producing the ARE at $1.6 \mathrm{GHz}$. The underlying red areas, delimited by the red square, highlight the middle-magnetosphere regions where the thermal plasma density is high enough to allow the ECM to mainly amplify the O-mode.

density given by the condition for the $1.6 \mathrm{GHz} \mathrm{O}$-mode amplification. These estimates are in a very good agreement with the EM derived from the analysis of X-ray observations. In fact, the hottest thermal plasma component observed in the $\rho$ OphC X-ray spectrum has $E M \approx 9 \times 10^{52} \mathrm{~cm}^{-3}$, derived using the relation $E M=$ $4 \pi d^{2} N \times 10^{14}$, where $d$ is the stellar distance and the normalization factor $N=4.2 \times 10^{-4} \mathrm{~cm}^{-5}$ (Pillitteri et al. 2016). Comparing the results retrieved by the radio and $\mathrm{X}$-ray observations, we can constraint the size of the non-thermal acceleration site. In fact, the measured $E M$ is almost equal to the estimated lower limit retrieved assuming a large middle-magnetosphere thickness. Therefore, a limited linear extension for the acceleration site of the nonthermal electrons is more reasonable.

\section{SUMMARY}

In this paper we report the detection of auroral radio emission from the early-type magnetic star $\rho$ OphC. Among this class, $\rho$ OphC is the seventh where the stellar ARE has been detected, but the first where the ARE was visible along the whole stellar rotation period.

The ARE is produced by the coherent ECM mechanism, that is characterized by a strongly anisotropic emission beam almost perpendicularly oriented with respect to the magnetic field vector. The ARE is detectable when the line of sight is almost perpendicular to the magnetic field axis. The permanent visibility of the ARE from $\rho$ OphC is a consequence of its ORM geometry. In fact, a dipole magnetic axis almost aligned with the stellar rotation axis, that in turn has a large inclination angle with the line-of-sight, is able to explain the observations.

In summary, the theoretical analysis of the ARE visibility condition enables us to constraint the ORM geometry of $\rho$ OphC. Further, we highlight the magneto-ionic O-mode mainly amplified by the ECM mechanism, a condition that constrains the density of the local thermal plasma. Finally, the available observations of $\rho$ OphC allow us to find out the contribution of the auroral thermal X-ray emission to the stellar X-ray spectrum.

As a general remark, the present paper confirms that combining observations at the extrema of the electromagnetic spec- trum (radio and X-ray) enable ones to efficiently investigate the plasma process occurring within the magnetospheres of the earlytype magnetic stars.

\section{ACKNOWLEDGMENTS}

We sincerely thank the anonymous referee for his/her very useful and constructive comments and suggestions. This work has extensively used the NASA's Astrophysics Data System, and the SIMBAD database, operated at CDS, Strasbourg, France. LMO acknowledges support from the DLR under grant FKZ 50 OR 1809 and partial support by the Russian Government Program of Competitive Growth of Kazan Federal University. JK was supported by grant GA ČR 18-05665S. FL was supported by "Programma ricerca di ateneo UNICT 2020-22 linea 2".

\section{APPENDIX A: DATA AVAILABILITY}

The data underlying this article are available in the article and in its online supplementary material.

\section{REFERENCES}

Alecian E. et al., 2014, A\&A, 567, 28

Babcock H. W., 1949, Observatory, 69, 191

Babel J., Montmerle T., 1997, A\&A, 323, 121

Badman S. V. et al., 2015, Space Sci. Rev., 187, 99

Bailey J. D. et al., 2012, MNRAS, 423, 328

Das B., Chandra P., Wade G. A., 2018, MNRAS, 474, L61

Das B., Chandra P., Shultz M. E., Wade G. A., 2019a, ApJ, 877, 123

Das B., Chandra P., Shultz M. E., Wade G. A., 2019b, MNRAS, 489, L102

Drake S. A. et al., 1987, ApJ, 322, 902

Gaia Collaboration et al., 2018, A\&A, 616, A1

Jilinski E., Daflon S., Cunha K., de La Reza R., 2006, A\&A, 448, 1001

Leone F., 1991, A\&A, 252, 198

Leone F., Trigilio C., Umana G., 1994, A\&A, 283, 908

Leto P. et al., 2006, A\&A, 458, 831

Leto P. et al., 2012, MNRAS, 423, 1766

Leto P. et al., 2016, MNRAS, 459, 1159

Leto P. et al., 2017, MNRAS, 467, 2820

Leto P. et al., 2018, MNRAS, 476, 562

Leto P. et al., 2019, MNRAS, 482, L4

Leto P. et al., 2020, MNRAS, 493, 4657

Linsky J. L., Drake S. A., Bastian S. A., 1992, ApJ, 393, 341

Louarn P., Le Queau D., 1996, P\&SS, 44, 211

Melrose D. B., Dulk G. A., 1982, ApJ, 259, 844

Melrose D. B., Hewitt R. G., Dulk G. A., 1984, J. Geophys. Res., 89, 897

Nazé Y. et al., 2014, ApJSS, 215, 10

Oskinova L. M. et al., 2011, MNRAS, 416, 1456

Pillitteri I. et al., 2016, A\&A, 592, 88

Pillitteri I. et al., 2017, A\&A, 602, 92

Rebull L. M., et al., 2018, ApJ, 155, 196

Robrade J. et al., 2018, A\&A, 619, 33

Sharma R. R., Vlahos L., 1984, ApJ, 280, 405

Shore S. N., Adelman S. J., 1981, LIACo, 23, 429

Stibbs D. W. N., 1950, MNRAS, 110, 395

Trigilio C. et al., 2000, A\&A, 362, 281

Trigilio C. et al., 2004, A\&A, 418, 593

Trigilio C. et al., 2008, MNRAS, 384, 1437

Trigilio C. et al., 2011, ApJ, 739, L10

Trigilio C. et al., 2018, MNRAS, 481, 217

Wu C. S., Lee L. C., 1979, ApJ, 230, 621

Zarka P., 1998, J. Geophys. Res., 103, 20159 